\title{
Long-term quality of life after surgery for adenocarcinoma of the esophagogastric junction: extended gastrectomy or transthoracic esophagectomy?
}

\author{
Hans Fuchs • Arnulf H. Hölscher • Jessica Leers • \\ Marc Bludau • Sebastian Brinkmann · Wolfgang Schröder • \\ Hakan Alakus • Stefan Mönig • Christian A. Gutschow
}

Received: 30 September 2014/ Accepted: 8 January 2015/Published online: 28 January 2015

(c) The International Gastric Cancer Association and The Japanese Gastric Cancer Association 2015

\begin{abstract}
Background Esophagectomy with gastric tube reconstruction and extended transhiatal gastrectomy with Rouxen-Y reconstruction are alternative procedures in current therapeutic concepts for adenocarcinoma of the esophagogastric junction (AEG). The impact of these operations on long-term health-related quality of life (HRQL) is incompletely understood.

Methods Patients with cancer-free survival of at least 24 months after esophagectomy (ESO) or extended gastrectomy (GAST) for AEG were identified from a prospectively maintained database. EORTC questionnaires were sent out to assess health-related general (QLQ-C30) and cancer-specific (OG-25) quality of life. Numeric scores were calculated for each conceptual area and compared with those of healthy reference populations.

Results 123 patients (ESO $n=71$; GAST $n=52$ ) completed the self-rated questionnaires. HRQL was consistently lower in surgical patients (GAST and ESO) compared with healthy reference populations. Also, there was a general trend for a better HRQL in GAST compared with ESO patients. This trend was statistically significant for physical function $(p=0.04)$, dyspnea $(p=0.02)$, and reflux $(p=0.03)$. Subgroup analysis revealed no significant differences between patients with or without prior neoadjuvant therapy.
\end{abstract}

H. Fuchs · A. H. Hölscher - J. Leers · M. Bludau •

S. Brinkmann · W. Schröder · H. Alakus · S. Mönig ·

C. A. Gutschow $(\square)$

Department of General-, Visceral-, and Cancer Surgery,

University of Cologne, Kerpener Strasse 62, 50931 Cologne,

Germany

e-mail: christian.gutschow@uk-koeln.de
Conclusions After mid- and long-term follow-up, HRQL after extended gastrectomy with Roux-en-Y reconstruction is superior to that after esophagectomy and gastric tube reconstruction. Improved HRQL after gastrectomy is mainly due to less pulmonary and reflux-related symptoms. Our findings may influence the choice of the surgical strategy for patients with AEG.

Keywords Esophagogastric junction cancer · Surgery · Quality of life

\section{Introduction}

The prognosis of patients with adenocarcinoma of the esophagogastric junction (AEG) is generally limited. However, along with the introduction of new multimodal treatment concepts during the last two decades, long-term survival in up to $55 \%$ of patients has been reported in surgical series from dedicated centers [1, 2]. Although cured from cancer, these patients often experience considerable therapy-related impairment of quality of life (HRQL) [3-6]. Among other factors, the choice of the surgical procedure may have an important impact on postoperative well-being. For Siewert type II and small Siewert type I carcinomas of the esophagogastric junction, extended gastrectomy with D2 lymphadenectomy including distal esophageal resection and reconstruction with a long jejunal loop according to Roux-en-Y in the lower mediastinum is typically performed. As an alternative, these tumors can be resected by transthoracic esophagectomy with radical two-field lymphadenectomy and reconstruction with a gastric pull-up procedure. From an oncological point of view, both strategies are equally 
justified by the topography of the lesions and their corresponding lymphatic drainage [7].

The purpose of this study was to compare long-term HRQL in patients that underwent one of the surgical procedures described above for adenocarcinoma of the esophagogastric junction.

\section{Materials and methods}

Patients and therapeutic concepts

643 patients underwent curative (R0) surgery for carcinoma of the esophagogastric junction in our department between June 1996 and December 2008. In this population, a transthoracic esophagectomy with radical two-field lymphadenectomy and reconstruction with a gastric pull-up procedure (ESO) was done in 366 patients, and an extended gastrectomy with D2 lymphadenectomy and resection of the distal esophagus and anastomosis in the lower mediastinum (GAST) was performed in 277 patients.

250 ESO and 107 GAST mid- to long-term survivors with a follow-up of at least 24 months were identified from a prospectively maintained database. All patients were free of recurrence and under active surveillance with annual computed tomography and endoscopy. Questionnaires were sent out to all patients of the ESO and GAST groups. Patients were instructed to fill in the questionnaires by themselves. 170 patients responded with a complete set of QOL data. Of these patients, 47 were excluded due to the following reasons: cervical anastomosis $(n=11)$, colonic interposition $(n=8)$, incomplete questionnaire $(n=3)$, and squamous cell carcinoma $(n=25)$. Clinical details of the remaining 123 study patients are summarized in Table 1.

36 of 123 patients ( $29 \%$ ) received neoadjuvant therapy before surgery. Neoadjuvant treatment was generally recommended for patients with advanced local tumor growth. Neoadjuvant therapies were done according to standardized recommendations that have been described in detail elsewhere [8].

All patients undergoing esophagectomy underwent a standardized Ivor-Lewis procedure that has been described in detail elsewhere $[9,10]$. The abdominal part of the operations (abdominal lymphadenectomy and gastrolysis) was done laparoscopically in 50 patients, and via laparotomy in 21 patients.

All patients undergoing extended gastrectomy underwent a standard open surgical procedure including D2lymphadenectomy and reconstruction with a long jejunal segment according to Roux-en-Y.

All patients fulfilling the criteria for HRQL assessment had an uneventful perioperative course without anastomostic leakage or endoscopic evidence of stenosis. This study was approved by our institutional review board.

\section{Quality of life assessment}

HRQL data was collected through the validated EORTC core questionnaire (QLQ C-30) and the cancer-specific module (QLQ OG-25). Validation of the questionnaires has been described elsewhere [11-15]. The QLQ C-30 version 3.0 is a standardized questionnaire that was developed to assess quality of life in cancer patients. It includes nine multi-item scales: a Global Health Status (GHS) scale, five functional scales (physical, role, cognitive, emotional, and social functioning), three symptom scales (fatigue, pain, and nausea) and six single-item scales (dyspnea, insomnia, appetite loss, constipation, diarrhea, and financial difficulties). The module QLQ OG-25 is designed to document esophageal cancer-specific symptoms and side effects. Its 25 questions transform into one functional scale (body image), six multi-item scales (dysphagia, problems with eating, reflux, odynophagia, pain and discomfort, and anxiety), and nine single-item scales (eating with others, dry mouth, trouble with taste, trouble swallowing saliva, choking when swallowing, trouble with coughing, and trouble talking, weight loss, and hair loss). Questionnaires
Table 1 Selected characteristics of the 71 patients treated with Ivor-Lewis esophagectomy (ESO) and the 52 patients treated with extended gastrectomy (GAST)

The Union internationale contre le cancer (UICC) classification was used for tumor staging

\begin{tabular}{lllr}
\hline & ESO $(n=71)$ & GAST $(n=52)$ & \multicolumn{1}{c}{$p$} \\
\hline Mean age at FU (years, range) & $67(38-87)$ & $73(22-91)$ & $<0.05$ \\
Follow-up (years, range) & $5.6(3-15)$ & $7.5(2.5-13.5)$ & $<0.05$ \\
Sex ratio (M:F) & $62: 9$ & $36: 16$ & $<0.05$ \\
UICC 0 & $7(9.9 \%)$ & $0(0 \%)$ & n.s. \\
UICC I & $47(66.2 \%)$ & $22(42.3 \%)$ & $<0.05$ \\
UICC IIa & $7(9.9 \%)$ & $5(9.6 \%)$ & n.s. \\
UICC IIb & $6(8.5 \%)$ & $7(13.5 \%)$ & n.s. \\
UICC III & $3(4.2 \%)$ & $10(19.2 \%)$ & $<0.05$ \\
Neoadjuvant therapy & $28(39.4 \%)$ & $8(18.1 \%)$ & $<0.05$ \\
Laparoscopic gastrolysis & $50(70.4 \%)$ & n.a. & n.a. \\
\hline
\end{tabular}


were sent out and patients were asked to send them back after filling in all questions by themselves. Missing data in the returned questionnaires were completed by telephone interview. Scoring algorithms have been developed by the EORTC [16]. All answers were transformed linearly to scores ranging from 0 to 100 . Missing data were handled according to the EORTC scoring manual. Mean scores with standard deviations (SD) were calculated. For functional scores and global QOL, higher scores represent better function and QOL. In symptom scales, higher scores indicate worse symptoms. Prevalence of symptoms was graded into the categories "symptoms" and "no symptoms" based on a score of at least two ("a little") in any question within a scale or relating to a single item.

\section{Reference populations}

HRQL data obtained by the European Organization for Research and Treatment of Cancer (EORTC) QLQ C-30 were compared to a published reference from 7802 healthy volunteers (age ranging from 40 to 80 years; $52 \%$ males and $48 \%$ females) provided by the EORTC [11], and EORTC OG-25 data were compared to a published reference from 4910 healthy volunteers (age ranging from 40 to 79 years; $69.9 \%$ males and $30.1 \%$ females) [15].

Assessment of data and statistical analysis

All data collected were entered into an Excel spreadsheet (Microsoft Office $2000^{\circledR}$, Microsoft Corporation, Redmond, WA, USA). Statistical analysis was performed with Statistica ${ }^{\circledR}$ (StatSoft, Tulsa, OK, USA). Mann-Whitney, Kruskal-Wallis, and $\chi^{2}$ tests were used as appropriate. A $p$ value of $<0.05$ was considered statistically significant.

\section{Results}

Global health score, functional scales, symptom scales, and single items of the EORTC QLQ C-30 (Table 2; Fig. 1)

We found a general trend for a lower HRQL in surgical patients (GAST and ESO groups) compared with the published healthy reference [11]. For HRQL scores, this trend was statistically significant $(p<0.05)$ in all scales and items except for emotional function, cognitive function, and pain in the ESO group, and in all scales and items except for global health score, emotional function, cognitive function, pain, and constipation in the GAST group.

Likewise, the prevalence of symptoms was generally lower in the reference group compared with the surgical groups. This trend was statistically significant $(p<0.05)$ for all symptoms except fatigue, pain, and insomnia in the ESO group and fatigue, pain, dyspnea, insomnia, and constipation in the GAST group.

Comparing patients of the ESO and GAST groups, we found a uniform trend for superior HRQL scores and lower prevalences of symptoms in gastrectomy patients. This trend was statistically significant for the HRQL scores of physical function $(p=0.04)$ and dyspnea $(p=0.02)$.

Subgroup analysis revealed no significant differences between patients with or without neoadjuvant therapy (data not shown).

\section{Organ-specific QOL of the EORTC OG-25 (Table 3)}

We found a general trend for a lower HRQL in surgical patients (GAST and ESO groups) compared with the published healthy reference [15]. For HRQL scores, this trend was statistically significant $(p<0.05)$ in all scales and items except for body image, trouble with taste, and trouble with talking in the ESO group, and in all scales and items except for trouble with taste and trouble with talking in the GAST group.

Likewise, the prevalence of symptoms was generally lower in reference individuals compared with the surgical groups. This trend was statistically significant $(p<0.05)$ for all symptoms except problems with eating and anxiety in the ESO group and problems with eating, reflux, anxiety, trouble with taste, trouble with coughing, and trouble with talking in the GAST group.

In contrast, there was no consistent trend for superior HRQL scores or lower prevalences of symptoms after extended gastrectomy compared with patients after esophagectomy and gastric tube reconstruction. However, after esophagectomy, a significantly higher score indicating a reduced HRQL was found for reflux $(p=0.03)$.

Subgroup analysis revealed no significant differences between patients with or without neoadjuvant therapy (data not shown).

\section{Discussion}

Since its introduction in 1987 [17-19], Siewert's classification of adenocarcinoma of the esophagogastric junction (AEG) has become a standard clinical tool to guide therapeutic decision making. In Siewert type I, the center of the tumor mass is located between 5 and $1 \mathrm{~cm}$ above the $z$-line, in type II from $1 \mathrm{~cm}$ above to $2 \mathrm{~cm}$ below the $z$-line, and in type III tumors $2-5 \mathrm{~cm}$ below this anatomical landmark. There is general agreement that AEG I should be treated with subtotal esophagectomy and resection of the proximal stomach, whereas patients with AEG III should undergo extended gastrectomy with transhiatal resection of the 
Table 2 Global health score (GHS), functional scales, and symptom scales of the EORTC QLQ C-30 in patients treated with esophagectomy (ESO) and extended gastrectomy (GAST) compared with normal values provided by the EORTC [11]

\begin{tabular}{|c|c|c|c|c|c|c|c|c|c|}
\hline & \multicolumn{2}{|c|}{$\mathrm{ESO}(n=70)$} & \multicolumn{2}{|c|}{ GAST $(n=50)$} & \multicolumn{2}{|c|}{ Normal value $(n=7802)$} & \multicolumn{3}{|l|}{$p$ values } \\
\hline & $\begin{array}{l}\text { Mean } \\
\text { score }\end{array}$ & $\begin{array}{l}\text { Symptoms } \\
(\%)^{\mathrm{a}}\end{array}$ & $\begin{array}{l}\text { Mean } \\
\text { score }\end{array}$ & $\begin{array}{l}\text { Symptoms } \\
(\%)^{\mathrm{a}}\end{array}$ & $\begin{array}{l}\text { Mean } \\
\text { score }\end{array}$ & $\begin{array}{l}\text { Symptoms } \\
(\%)^{\mathrm{a}}\end{array}$ & ESO/GAS & ESO/normal & GAS/normal \\
\hline GHS & $60.0(21.2)$ & na & $65.5(23.6)$ & na & $71.2(22.4)$ & na & ns & $<0.05$ & ns \\
\hline Physical function & $73.7(19.2)$ & na & $79.7(20.5)$ & na & $89.8(16.2)$ & na & $<0.05$ & $<0.05$ & $<0.05$ \\
\hline Role function & $64.8(30.2)$ & na & $70.0(29.9)$ & na & $84.7(25.4)$ & na & ns & $<0.05$ & $<0.05$ \\
\hline Emotional function & $64.2(29.0)$ & na & $68.0(30.0)$ & na & $76.3(22.8)$ & na & ns & ns & ns \\
\hline Cognitive function & $78.1(25.8)$ & na & $77.0(27.1)$ & na & $86.1(20.0)$ & na & $\mathrm{ns}$ & ns & ns \\
\hline Social function & $63.1(29.5)$ & na & $71.3(33.7)$ & na & $87.5(22.9)$ & na & ns & $<0.05$ & $<0.05$ \\
\hline Fatigue & $42.2(28.0)$ & $61(85.9)$ & $35.6(29.3)$ & $40(80.0)$ & $24.1(24.0)$ & na & ns & $<0.05$ & $<0.05$ \\
\hline Nausea & $15.2(21.4)$ & $34(48.6)$ & $18.3(33.5)$ & $17(34.0)$ & $3.7(11.7)$ & na & $\mathrm{ns}$ & $<0.05$ & $<0.05$ \\
\hline Pain & $29.0(31.0)$ & $61(87.1)$ & $24.7(31.3)$ & $39(78.0)$ & $20.9(27.6)$ & na & ns & ns & ns \\
\hline Dyspnea & $44.8(33.5)$ & $52(74.3)$ & $24.7(30.7)$ & $22(44.0)$ & $11.8(22.8)$ & $1946(25.2)$ & $<0.05$ & $<0.05$ & $<0.05$ \\
\hline Insomnia & $38.6(31.4)$ & $49(70.0)$ & $36.0(34.2)$ & $30(60.0)$ & $21.8(29.7)$ & $3280(42.3)$ & ns & $<0.05$ & $<0.05$ \\
\hline Appetite & $21.0(29.0)$ & $28(40.0)$ & $18.0(28.7)$ & $16(32.0)$ & $6.7(18.3)$ & $1117(14.4)$ & $\mathrm{ns}$ & $<0.05$ & $<0.05$ \\
\hline Constipation & $16.7(25.9)$ & $24(34.3)$ & $12.0(23.1)$ & $13(26.0)$ & $6.7(18.4)$ & 1104 (14.3) & ns & $<0.05$ & ns \\
\hline Diarrhea & $36.2(33.0)$ & $46(65.7)$ & 34.7 (33.6) & $30(60.0)$ & $7.0(18.0)$ & $1206(15.6)$ & ns & $<0.05$ & $<0.05$ \\
\hline Finances & 22.4 (33.9) & $26(37.1)$ & $22.7(33.3)$ & 19 (38.0) & $9.5(23.3)$ & 1346 (17.4) & ns & $<0.05$ & $<0.05$ \\
\hline
\end{tabular}

Data are expressed in means (standard deviation) and prevalence of symptoms (\%)

" Patients who answered "a little," "quite a bit," or "very much" to any question within a scale or to a single item na not applicable, $n s$ not significant

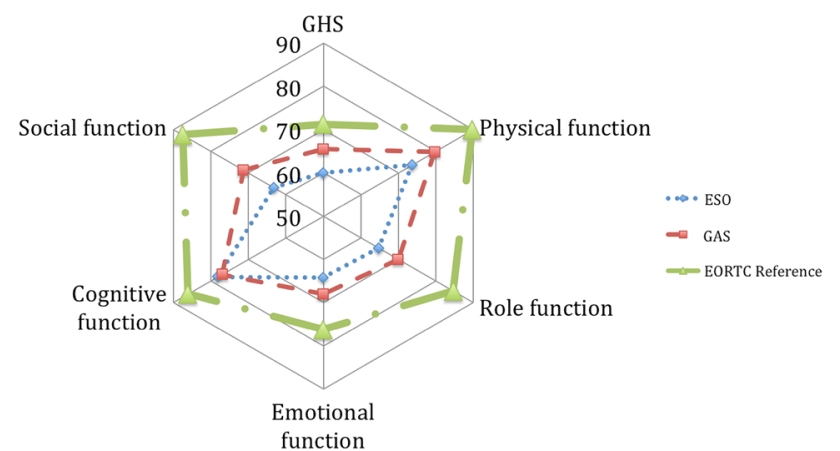

Fig. 1 Spider graph representing mean scores for global health (GHS) and functional scales of the EORTC QLQ C-30 in patients after Ivor-Lewis esophagectomy (squares), after extended gastrectomy (triangles), and in the EORTC reference population [11]

distal esophagus [20]. In contrast, there is uncertainty regarding the ideal therapeutic strategy for AEG type II. In this specific situation, both procedures have been shown to be oncologically equivalent and to provide similar longterm survival rates $[1,7,20]$. As a consequence, most current guidelines recommend performing either esophagectomy with resection of the proximal stomach and reconstruction with a gastric tube or, as an alternative, extended gastrectomy with transhiatal resection of the lower esophagus and reconstruction with a Roux-en-Y jejunal limb [8]. It is evident that the diagnosis of AEG has a significant influence on general and organ-specific HRQL. Curative surgery generally aims at healing the patient and re-establishing HRQL as complete as possible. In this specific entity, these goals are difficult to achieve due to the oncological aggressiveness of the disease, due to the complexity of the surgical interventions (with a considerable risk of morbidity and mortality), and due to the inevitable surgical mutilation of the patient (with considerable postoperative changes of the foregut anatomy and subsequent functional deficits).

The published evidence on mid- and long-term HRQL after esophagectomy and gastrectomy is controversial. From other research, we know that significant recuperation of HRQL takes place during the first 1-2 years [4, 14], and recovery to levels similar to that of a healthy reference population has been documented in a number of studies [21-25]. Likewise, we could demonstrate in an earlier trial that $50 \%$ of mid- and long term survivors of the IvorLewis procedure for esophageal cancer came out with a HRQL similar to that of a healthy reference population [26]. However, significantly reduced long-term HRQL as compared with matched reference populations has also been described [5, 27-29]. Our study confirms the latter hypothesis that long-term HRQL after major upper-GI surgery remains reduced in a number of aspects, and we must accept that complete recovery did not take place in the majority of patients. 
Table 3 Symptom scales of the EORTC OG-25 treated with esophagectomy (ESO) and extended gastrectomy (GAST) compared with published normal values [15]

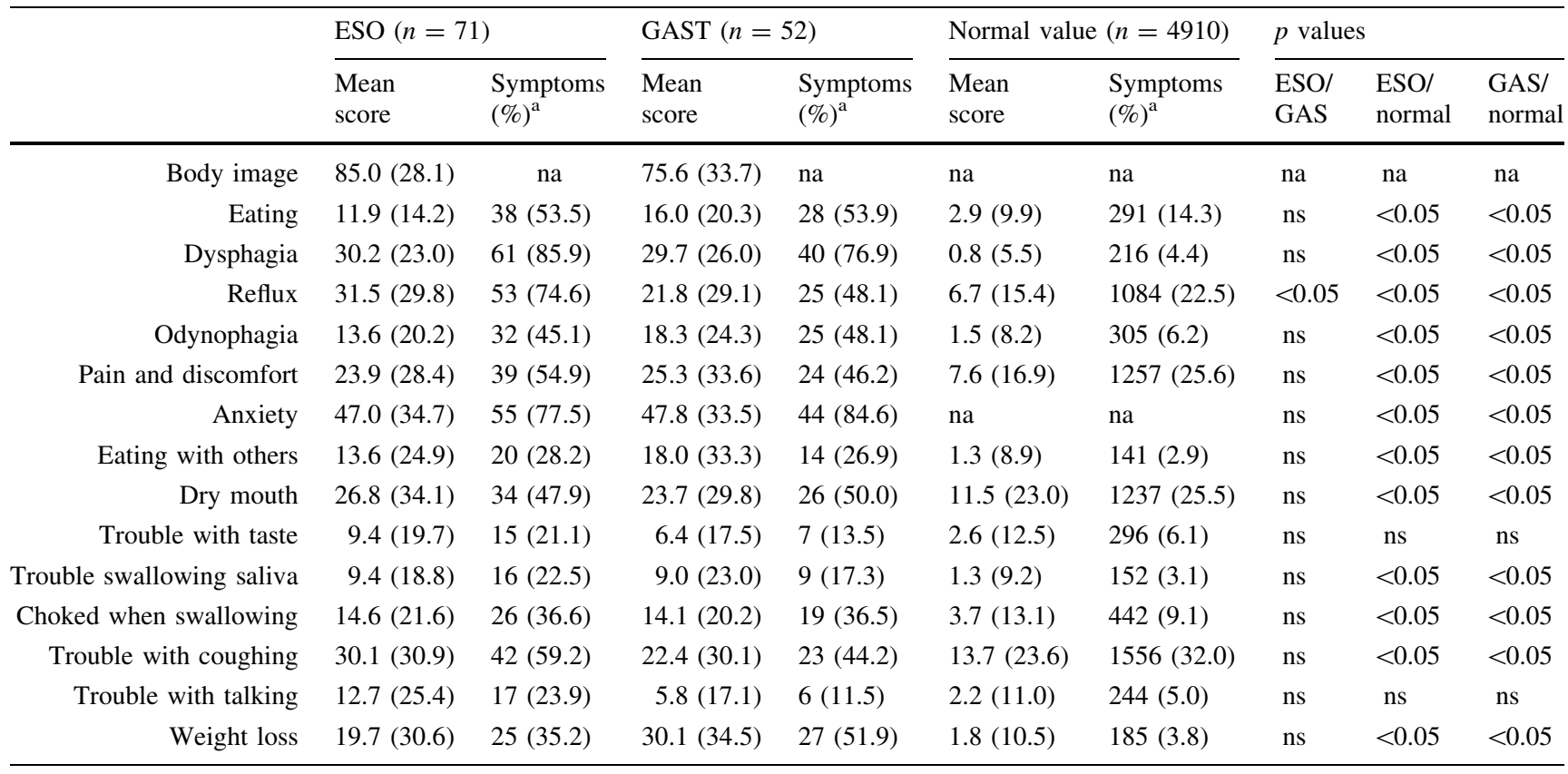

Data are expressed in means (standard deviation) and prevalence of symptoms (\%)

a Patients who answered "a little," "quite a bit," or "very much" to any question within a scale or to a single item

On the other hand, our study is the first to provide a direct comparison of long-term HRQL data in AEG patients after extended gastrectomy with Roux-en-Y reconstruction and transthoracic esophagectomy with gastric tube reconstruction. We were able to show that HRQL after gastrectomy is superior to that after esophagectomy. Impaired long-term HRQL after esophagectomy was mainly due to increased pulmonary and reflux-related symptoms. Continuous higher levels of dyspnea in the ESO group even 2 years after the operation might still be due to pulmonary adhesions after the thoracic part of the IvorLewis esophagectomy. From our earlier research, we know that the intraluminal acidity of the denervated gastric tube-used as an esophageal substitute-normalizes over time: more than 3 years after the operation, the $24-\mathrm{h} \mathrm{pH}$ profile in the gastric cavity in most patients is similar to that in healthy subjects [30]. The latter phenomenon is associated with a rising incidence of gastroesophageal reflux disease, macroscopic esophagitis, metaplasia, and the need for a potent anti-reflux medication [31, 32]. Thus, we can conclude from our study that reflux-related symptoms are the main cause of postoperative reduction of HRQL in esophagectomized patients. Another finding of interest is that patients in the GAS group showed better performance despite their higher ages. This underlines our assumption that extended total gastrectomy has a reduced impact on postoperative HRQL than esophagectomy and is therefore a valuable option, even in older patients.

In a recent study covering the same scientific issue, a comparison of the short-term (6 months) HRQL after gastrectomy and esophagectomy for AEG was performed using the EORTC QLQ C-30 questionnaire. As in our study, a greater negative impact on HRQL after esophagectomy compared with gastrectomy was found, and the authors concluded that gastrectomy has the potential to reduce the functional side effects of major upper-GI surgery [33].

Our study has a number of potential limitations. First, we cannot exclude a selection bias due to the retrospective design of our trial. However, $48 \%$ of the patients responded with a complete set of data and, as no selection was performed and loss to follow-up occurred at random, we consider the patients to be representative of the complete patient group. Also, there is an inevitable bias due to the rather high death rate among AEG patients. Another relevant problem may be the fact that we were not able to compare our data to pretreatment baseline HRQL. On the other hand, we were able to analyze two highly selective rather homogeneous groups of patients that underwent standardized surgical procedures. In fact, the surgical access to the abdominal cavity for gastrolysis (laparoscopy $n=50$; laparotomy $n=21$ ) was the only relevant 
variable. Also, the follow-up was long enough to exclude immediate side effects of the surgical intervention during the early recovery phase.

In summary, we can conclude from our study that extended total gastrectomy provides a better functional outcome than esophagectomy. Therefore, gastrectomy should be the preferred surgical option for AEG type II according to Siewert's classification. Our findings may play a significant role in the choice of surgical strategy for patients with this oncologic entity.

Conflict of interest No funding obtained, no conflicts of interest.

\section{References}

1. Curtis NJ, Noble F, Bailey IS, Kelly JJ, Byrne JP, Underwood TJ. The relevance of the Siewert classification in the era of multimodal therapy for adenocarcinoma of the gastro-oesophageal junction. J Surg Oncol. 2014;109:202-7.

2. Vallböhmer D, Hölscher AH, DeMeester S, DeMeester T, Salo J, Peters J, et al. A multicenter study of survival after neoadjuvant radiotherapy/chemotherapy and esophagectomy for ypT0NOM0R0 esophageal cancer. Ann Surg. 2010;252:744-9.

3. Lagergren P, Avery KN, Hughes R, Barham CP, Alderson D, Falk SJ, et al. Health-related quality of life among patients cured by surgery for esophageal cancer. Cancer. 2007;110:686-93.

4. Viklund P, Lindblad M, Lagergren J. Influence of surgery-related factors on quality of life after esophageal or cardia cancer resection. World J Surg. 2005;29:841-8.

5. Gockel I, Gönner U, Domeyer M, Lang H, Junginger T. Long-term survivors of esophageal cancer: disease-specific quality of life, general health and complications. J Surg Oncol. 2010;102:516-22.

6. Bollschweiler E, Baltin C, Berlth F, Mönig SP, Hölscher AH. Quality of life and visceral surgery. Chirurg. 2014;85:203-7.

7. Siewert RJ, Feith M, Werner M, Stein HJ. Adenocarcinoma of the esophagogastric junction: results of surgical therapy based on anatomical/topographic classification in 1,002 consecutive patients. Ann Surg. 2000;232:353-61.

8. Moehler M, Al-Batran SE, Andus T, Anthuber M, Arends J, Arnold D, et al. German S3-guideline diagnosis and treatment of esophagogastric cancer. Z Gastroenterol. 2011;49:461-531.

9. Lewis I. The surgical treatment of carcinoma of the oesophagus; with special reference to a new operation for growths of the middle third. Br J Surg. 1946;34:18-31.

10. Hölscher AH, Schneider PM, Gutschow C, Schröder W. Laparoscopic ischemic conditioning of the stomach for esophageal replacement. Ann Surg. 2007;245:241-6.

11. Scott NW, Fayers PM, Aaronson NK et al. EORTC QLQ-C 30 reference values. http://groups.eortc.be/qol/downloads/reference_ values_manual2008.pdf. Accessed 21 Nov 2014.

12. Aaronson NK, Ahmedzai S, Bergman B, Bullinger M, Cull A, Duez NJ, et al. The European Organization for Research and Treatment of Cancer QLQ-C30: a quality-of-life instrument for use in international clinical trials in oncology. J Natl Cancer Inst. 1993;85:365-76.

13. Blazeby JM, Alderson D, Winstone K, Steyn R, Hammerlid E, Arraras J, et al. Development of an EORTC questionnaire module to be used in quality of life assessment for patients with oesophageal cancer. The EORTC Quality of Life Study Group. Eur J Cancer. 1996;32A:1912-7.
14. Fayers P, Aaronson NK, Bjordal K, Groenvold M, Curran D, Bottomley A. EORTC QLQ-C 30 scoring manual. 3rd ed. Brussels: European Organisation for Research and Treatment of Cancer; 2001.

15. van der Schaaf M, Derogar M, Lagergren P. Reference values of oesophago-gastric symptoms (EORTC QLQ-OG25) in a population-based setting. Eur J Cancer. 2012;48:1602-7.

16. Fayers PM, Machin D. Quality of life: assessment, analysis and interpretation. Chichester: John Wiley \& Sons; 2007.

17. Siewert JR, Hölscher AH, Becker K, Gössner W. Cardia cancer: attempt at a therapeutically relevant classification. Chirurg. 1987;58:25-32.

18. Siewert JR, Stein HJ. Adenocarcinoma of the gastroesophageal junction: classification, pathology and extent of resection. Dis Esophagus. 1996;9:173-82.

19. Siewert JR, Stein HJ. Classification of adenocarcinoma of the oesophagogastric junction. Br J Surg. 1998;85:1457-9.

20. Feith M, Stein HJ, Siewert JR. Adenocarcinoma of the esophagogastric junction: surgical therapy based on 1602 consecutive resected patients. Surg Oncol Clin N Am. 2006;15:751-64.

21. McLarty AJ, Deschamps C, Trastek VF, Allen MS, Pairolero PC, Harmsen WS. Esophageal resection for cancer of the esophagus: long-term function and quality of life. Ann Thorac Surg. 1997;63:1568-72.

22. De Boer AG, Genovesi PI, Sprangers MA, Van Sandick JW, Obertop H, Van Lanschot JJ. Quality of life in long-term survivors after curative transhiatal oesophagectomy for oesophageal carcinoma. Br J Surg. 2000;87:1716-21.

23. Deschamps C, Nichols FC 3rd, Cassivi SD, Allen MS, Pairolero PC. Long-term function and quality of life after esophageal resection for cancer and Barrett's. Surg Clin North Am. 2005;85:649-56.

24. Moraca RJ, Low DE. Outcomes and health-related quality of life after esophagectomy for high-grade dysplasia and intramucosal cancer. Arch Surg. 2006;141:545-9 (discussion 549-51).

25. Karanicolas PJ, Graham D, Gönen M, Strong VE, Brennan MF, Coit DG. Quality of life after gastrectomy for adenocarcinoma: a prospective cohort study. Ann Surg. 2013;257:1039-46.

26. Gutschow CA, Hölscher AH, Leers J, Fuchs H, Bludau M, Prenzel KL, et al. Health-related quality of life after Ivor Lewis esophagectomy. Langenbecks Arch Surg. 2013;398:231-7.

27. Djärv T, Lagergren J, Blazeby JM, Lagergren P. Long-term health-related quality of life following surgery for oesophageal cancer. Br J Surg. 2008;95:1121-6.

28. Donohoe CL, McGillycuddy E, Reynolds JV. Long-term healthrelated quality of life for disease-free esophageal cancer patients. World J Surg. 2011;35:1853-60.

29. Kim AR, Cho J, Hsu YJ, Choi MG, Noh JH, Sohn TS, et al Changes of quality of life in gastric cancer patients after curative resection: a longitudinal cohort study in Korea. Ann Surg. 2012;256:1008-13.

30. Gutschow C, Collard JM, Romagnoli R, Salizzoni M, Hölscher A. Denervated stomach as an esophageal substitute recovers intraluminal acidity with time. Ann Surg. 2001;233:509-14.

31. Wolfsen HC, Hemminger LL, DeVault KR. Recurrent Barrett's esophagus and adenocarcinoma after esophagectomy. BMC Gastroenterol. 2004;4:18.

32. Gutschow CA, Vallböhmer D, Stolte M, Oh D, Danenberg K, Danenberg $\mathrm{P}$, et al. Adenocarcinoma developing in de novo Barrett's mucosa in the remnant esophagus after esophagectomy: clinical and molecular assessment. Dis Esophagus. 2008;21:E6-8.

33. Barbour AP, Lagergren P, Hughes R, Alderson D, Barham CP, Blazeby JM. Health-related quality of life among patients with adenocarcinoma of the gastro-oesophageal junction treated by gastrectomy or oesophagectomy. Br J Surg. 2008;95:80-4. 